\title{
Model-based Optimal Sensor Placement Approaches to Fuel Cell Stack System Fault Diagnosis *
}

\author{
Ramon Sarrate $^{*}$ Fatiha Nejjari * Albert Rosich ${ }^{* *}$ \\ * Automatic Control Department, Universitat Politècnica de Catalunya \\ (UPC), Rambla de Sant Nebridi, 11, 08222 Terrassa, Spain. \\ ** Institute of Robotics and Industrial Informatics, Edifici U, Pau Gargallo, 5, \\ 08028-Barcelona, Spain
}

\begin{abstract}
The problem of optimal sensor placement for FDI consists in determining the set of sensors that minimizes a pre-defined cost function satisfying at the same time a pre-established set of FDI specifications for a given set of faults. This paper recalls three model-based optimal sensor location approaches: an Incremental search, a Heuristic search and a Binary Integer Linear Programming (BILP) formulation. The main contribution of this paper is a comparative study that addresses efficiency, flexibility and other issues. The performance of the approaches is demonstrated by an application to a fuel cell stack system.
\end{abstract}

Keywords: Optimal Sensor Placement, Fault Detection and Isolation, Fuel Cell Stack System

\section{INTRODUCTION}

The performance of an industrial process is strongly dependent on available sensor measurements. Inaccurate measurements resulting from insufficient measurements or improper sensor placement can significantly deteriorate fault observability and process control.

The problem of sensor placement for Fault Detection and Isolation (FDI) consists in determining the optimal set of instruments such that a predefined set of faults are detected and isolated. The usual objective to minimize in the sensor placement problem is the sensor cost. The sensor placement problem can then be viewed as a combinatorial problem that consists in finding a sensor combination that fulfils diagnosis specifications.

Solving the sensor placement for diagnosis can be treated from many different points of view. Indeed, such a problem depends on the kind of system description, the required diagnosis specifications, as well as the technique used to implement the diagnosis system. Because of this, developing a sensor placement method, that works for all possible fault diagnosis systems, is unattainable. In this paper, fault diagnosis systems are based on consistency checking by means of structural models. The required diagnosis specifications to be fulfilled are fault detection and isolation for a predefined set of faults.

In the last decade, a lot of works have been devoted to analyze diagnosability and sensor placement in the context of modelbased diagnosis. In Travé-Massuyès et al. (2006), optimal sensor placement for model-based FDI requires to find the set of all possible analytical redundancy relations (ARRs), considering that all candidate sensors are installed. Then, a set of sensors

\footnotetext{
* This work has been funded by the Spanish Ministry of Science and Technology through the CICYT project WATMAN (ref. DPI2009-13744), by the Spanish Ministry of Economy and Competitiveness through the CICYT project SHERECS (ref. DPI2011-26243), and by the European Commission through contract i-Sense (ref. FP7-ICT-2009- 6-270428).
}

that minimizes the total cost of the network is selected such that the resulting ARRs satisfy that a pre-established set of faults can be detected and isolated. However, the associated computational complexity is exponential with the number of candidate sensors. In Rosich et al. (2007) this search strategy is modified by starting with no sensors and iteratively adding sensors while the Minimal Structurally Overdetermined (MSO) sets are incrementally generated. In Sarrate et al. (2007), an optimal sensor placement for model-based FDI requires finding the set of all possible MSO sets, considering that all candidate sensors are installed. The optimization problem is casted as a Binary Integer Programming problem (Wosley, 1998), where the optimization vector states whether a sensor is installed or not, and the FDI specifications are translated into constraints. However, the non-linear nature of such constraints lead to a high computational complexity of the resulting optimization problem. An alternative approach, which involves the formulation of a non-linear objective function, is proposed in Fijany and Vatan (2006). In Rosich et al. (2009) and Nejjari et al. (2010), the approach in Sarrate et al. (2007) is enhanced by formulating a Binary Integer Linear Programming (BILP) problem. The FDI specifications are formulated as linear constraints and the objective cost function is also linear, so that the BILP problem can be efficiently solved by an LP-based branch-and-bound algorithm.

On the other hand, generating the complete set of MSO sets requires a high computation time. To deal with this problem, some works have appeared recently without the requirement of computing any MSO set. These methods ensure that the MSO sets with the corresponding diagnosis capabilities can be generated after solving the sensor placement problem. In (Krysander and Frisk, 2008), an efficient method to find all the minimal sensors set ${ }^{1}$ for maximum fault detectability and isolabilty from a structural model is proposed. On the other

\footnotetext{
1 minimal set means that no proper subset is a solution.
} 
hand, in (Yassine et al., 2008) an alternative structural model decomposition, based on gathering equations that can not be isolable is proposed. From this decomposition, it is possible to determine the set of detectable faults and the set of isolable faults. Then, the optimal search on the candidate sensor set is performed. Finally, in (Rosich et al., 2012), a method that takes into account the causal computability of the unknown variables in the residual generation is developed.

This paper presents a comparative study of three modelbased optimal sensor placement approaches: a heuristic search (Rosich et al., 2010), an incremental algorithm (Rosich et al., 2007) and a Binary Integer Linear Programming formulation approach (Rosich et al., 2009) . The efficiency of these formulations is shown and compared by their application to a fuel cell system. A fuel cell system benchmark is used and some faults are defined to be diagnosed.

Fuel cell systems are receiving much attention in the last decade as good candidates for clean electricity generation. The fuel cell model involves a wide range of non-linear equations: lookup tables, piecewise functions, saturations, non-linear dynamic equations, etc. One way to analyze such a complex class of models in a general framework is considering its model structure. A structural model is a coarse model description, based on a bi-partite graph, that can be obtained early in the process development, without major engineering efforts.

In Section 2, the sensor placement problem is formulated. The three approaches to solve the sensor placement problem are briefly described in Sections 3-5. Section 6 describes the Fuel Cell Stack (FCS) system and presents the results obtained when solving the optimal sensor placement problem. In Section 7, the performance of the three approaches is discussed. Finally, some conclusions and remarks are given in Section 8.

\section{PROBLEM FORMULATION}

The aim of the sensor placement for fault diagnosis can be roughly stated as the choice of a sensor configuration such that a fault diagnosis specification is fulfilled. Usually, several sensor configurations that satisfy the required fault diagnosis specification will exist. Giving a cost to every sensor configuration makes it possible to define an optimal sensor placement problem, where the best sensor configuration is sought.

Let $\mathbf{S}$ be the set of candidate sensors. Any sensor $s \in \mathbf{S}$ can be chosen for installation, involving a cost denoted by $C(s)$. Such cost can comprise different concepts such as the purchase price, the maintenance price, the sensor reliability or the measurement precision, for instance. Given a sensor configuration $S \subseteq \mathrm{S}$, its cost will be defined as $\mathcal{C}(S) \triangleq \sum_{s \in S} C(s)$.

Let $\mathbf{F}$ be the set of faults that must be monitored. The diagnosis specifications will be stated based on two properties: fault detectability and fault isolability. In this work, the single fault assumption will hold (i.e., multiple faults will not be covered) and no candidate sensor fault will be considered.

A fault $f \in \mathbf{F}$ is detectable if its occurrence can be observed, whereas a fault $f_{i}$ is isolable from a fault $f_{j}$ if the occurrence of $f_{i}$ can be observed independently of the occurrence of $f_{j}$. A formal definition of these two properties can be found in Krysander (2006). The fault detectability specification will be defined as:

$$
F_{\mathcal{D}}=\{f \in \mathbf{F} \mid f \text { is detectable }\}
$$

whereas the fault isolability specification will be defined as $\mathbb{F}_{I}=\left\{\ldots, F_{\mathcal{I}}\left(f_{i}\right), \ldots\right\} \quad \forall f_{i} \in F_{\mathcal{D}}$, with:

$$
F_{\mathcal{I}}\left(f_{i}\right)=\left\{f_{j} \in F_{\mathcal{D}} \mid f_{i} \text { is isolable from } f_{j}\right\} .
$$

In this paper, a structural model will be used to solve the sensor placement problem. A structural model is formalized as a bipartite graph $G(M, X, A)$, where $M$ is a set of model equations, $X$ a set of unknown variables and $A$ a set of edges such that $\left(e_{i}, x_{j}\right) \in A$ as long as equation $e_{i} \in M$ depends on the variable $x_{j} \in X$. In the following, $\mathbf{M}$ will denote the set of equations describing the fault-free behaviour of the system.

Structural modelling is suitable for an early stage of the system design, when the precise model expressions are not known yet, but it is possible to determine which variables are related to each equation. Furthermore, the diagnosis analysis based on structural models are performed by means of graph-based methods which have no numerical problems and are more efficient, in general, than analytical methods.

The optimal sensor placement for fault diagnosis can be formally stated as follows:

GIVEN A set of candidate sensors $\mathbf{S}$, a sensor cost function $C(\cdot)$, a set of model equations $M$, a fault detectability specification $F_{\mathcal{D}}$ and a fault isolability specification $\mathbb{F}_{I}$,

FIND the minimum cost sensor configuration $S^{*} \subseteq \mathrm{S}$, such that the fault diagnosis specifications are fulfilled.

This problem involves solving the following two issues: a search strategy for a candidate solution to the optimal sensor placement problem, and the verification of the fault diagnosis specifications fulfilment of this candidate solution. The desired fault diagnosis specifications will be required to be satisfied when all candidate sensors are installed in the system.

There are two possible approaches to the verification of the fault diagnosis specifications for a given structural model: the verification based on the set of redundant sub-models and the verification based on structural model properties.

Redundant sub-models are of central importance in diagnosis. Several structural approaches to find redundant sub-models are suggested in the literature. The name given to redundant sub-models depends on the approach, e.g. analytical redundancy relations, ARR (Travé-Massuyès et al., 2006), minimal structurally overdetermined set, MSO (Krysander et al., 2008), testable sub-system, TSS (Ploix et al., 2008) and minimal evaluation chain, MEC (Pulido and Gonzalez, 2004). All these approaches have in common the fact that each redundant submodel has a complete matching in the unknown variables plus an extra equation, the redundant equation, used for checking consistency. In this paper, the MSO approach will be followed.

For a given sensor configuration $S$, the full set of MSO sets $\Omega_{S}$ can be determined using Algorithm MSO in (Krysander et al., 2008). Then, fault detectability and fault isolability can be verified based on the so-called Fault Signature Matrix $V$. Let $n$ be the number of MSO sets in $\Omega_{S}$ and $l$ be the number of faults in $\mathbf{F}$. The binary matrix $V$ of size $n \times l$ stores the sensitivity of an MSO set to the set of faults: if an MSO set $\omega_{i} \in \Omega_{S}$ is sensitive to fault $j$ then $v_{i j}=1$, otherwise $v_{i j}=0$. Then, a fault $f_{j} \in \mathbf{F}$ is detectable if there exists at least one MSO set $\omega_{i} \in \Omega_{S}$ such that $v_{i j}=1$. And a fault $f_{j_{1}}$ is isolable 
from a fault $f_{j_{2}}$ if there exists at least one MSO set $\omega_{i} \in \Omega_{S}$ such that $v_{i j_{1}}=1 \wedge v_{i j_{2}}=0$.

On the other hand, it is possible to verify the fault diagnosis specifications by applying the DM decomposition (Dulmage and Mendelsohn, 1958). The DM decomposition defines a partition on the set of equations and the set of unknown variables of the structural model. Three main parts of $M$ can be identified in the partition, namely, the under-determined part, the just-determined part and the over-determined part. In the over-determined part, there are more equations than unknown variables, which implies that there exists some degree of redundancy, and this is the part of the model that is useful for monitoring the process. In the following, the notation $\mathrm{M}^{+}$will represent the over-determined part of a given set of model equations $M$.

Now, the structural characterisation of the fault diagnosis properties of a system can be stated based on the set of model equations $M$ (Krysander and Frisk, 2008). Without loss of generality, it is assumed that a single fault $f \in \mathbf{F}$ can only violate one equation, denoted by $e_{f} \in M$. A fault $f \in F$ is detectable in a model $M$ if $e_{f} \in M^{+}$. And a fault $f_{i}$ is isolable from $f_{j}$ in a model $M$ if $e_{f_{i}} \in\left(M \backslash\left\{e_{f_{j}}\right\}\right)^{+}$.

The next three sections of the paper recall three different search strategies for a candidate solution for the optimal sensor placement problem. In the first one, the verification of the fault diagnosis specifications will be based on the DM decomposition of the structural model, whereas in the other two approaches the Fault Signature Matrix will be used.

\section{HEURISTIC APPROACH (Rosich et al., 2010)}

Algorithm 1 solves the search strategy. It is based on a depthfirst search by choosing first the nodes with lowest costs and back-tracking to other not already explored nodes when a branch exploration is aborted.

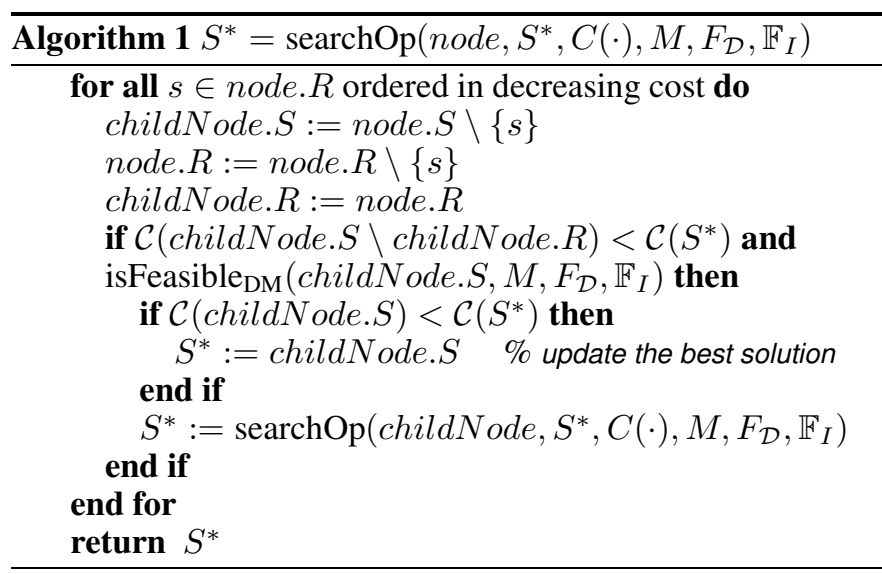

Every node in the tree consists of two sensor sets:

- node.S, the sensor configuration that the node represents (i.e., a candidate solution to test).

- node. $R$, the sensors that are allowed to be removed in its sub-nodes.

Throughout the search, the best solution is updated in $S^{*}$, whenever a feasible solution with lower cost than the current best one is found. A branch exploration is terminated at some node when any of the following two conditions is fulfilled:
Condition 1: The expected lowest reachable cost by exploring sub-nodes of the current node is not lower than the cost of the current best solution.

Condition 2: The node is not a feasible solution for the sensor placement problem.

In Algorithm 1, the boolean function isFeasible DM $_{\text {verifies the }}$ fault diagnosis specifications for a given sensor configuration. This function is implemented based on the DM decomposition approach recalled in Section 2.

Algorithm 1 is initialised with the candidate sensor set as the root node of the search tree, the candidate sensor set as the current best sensor configuration, the cost corresponding to the candidate sensors, the fault-free system model and the desired fault diagnosis specifications.

\section{BILP APPROACH (Rosich et al., 2009)}

A standard optimization problem using BILP can be formulated as a linear objective function and constrained by linear inequality constraints:

$$
\begin{aligned}
\min _{\mathbf{x}} \mathbf{c}^{T} \mathbf{x} \quad \text { subject to: } & \\
& \mathbf{A x} \leq \mathbf{b} \\
& \mathbf{x} \text { is binary }
\end{aligned}
$$

The main constraint is that any element of the optimization vector $\mathbf{x}$ must be binary, i.e. $\forall x \in \mathbf{x}: x \in\{0,1\}$. Furthermore, matrix $\mathbf{A}$ and vector $\mathbf{b}$ form the linear inequality constraints. Finally, $\mathbf{c}$ is a cost vector of the linear objective function.

The sensor placement problem can be formulated as a BILP problem where the set of candidate sensors to be installed is represented by an optimization variable vector $\mathbf{q}$. This means that if entry $q_{i} \in \mathbf{q}$ equals 1 , the corresponding sensor $s_{i} \in \mathbf{S}$ must be installed, whereas if $q_{i}$ equals 0 , the sensor does not need to be installed.

The main advantage of using this approach is that, once the constraints are formulated, standard branch and bound search strategies available in commercial optimization environments can be used.

This approach requires to formulate all constraints as linear inequalities. Thus, fault detectability and fault isolability specifications must be expressed as in (4).

The method requires the computation of the complete set of MSO sets since the fault diagnosis specifications will be formulated on the Fault Signature Matrix $V$. The complete set means assuming that all candidate sensors are installed, i.e. $\Omega_{\mathbf{S}}$.

In a sensor placement problem, it is important to remark that the set of MSO sets depends on the sensor configuration. Let $k$ be the number of sensors in $\mathbf{S}$. Then, a binary matrix $W$ of size $n \times k$, which describes this correspondence, is defined: if an MSO set $\omega_{i}$ depends on sensor $s_{j}$ then $w_{i j}=1$, otherwise $w_{i j}=0$.

The tight correspondence between an MSO set and a sensor configuration leads to the definition of the linear MSO set selector $\lambda$. Constraint (6) states that if an MSO set $\omega_{i}$ is not valid for a given sensor configuration then $\lambda_{i}=0$ :

$$
\sum_{j=1}^{k}\left[w_{i j} q_{j}+\left(1-w_{i j}\right)\right]-k \lambda_{i} \geq 0
$$


The fault detectability and isolability specifications can be formulated using the linear MSO set selector, based on the definitions given in Section 2. A fault $f_{j} \in \mathbf{F}$ is detectable if constraint (7) is satisfied.

$$
\sum_{i=1}^{n} v_{i j} \lambda_{i} \geq 1
$$

A fault $f_{j_{1}}$ is isolable from a fault $f_{j_{2}}$ if constraint (8) is satisfied.

$$
\sum_{i=1}^{n} v_{i j_{1}}\left(1-v_{i j_{2}}\right) \lambda_{i} \geq 1
$$

Once detectability and isolability constraints have been introduced, the optimal sensor placement for FDI can be formally stated as

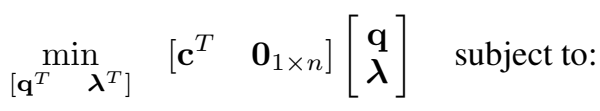

$$
\begin{aligned}
& \begin{array}{l}
\mathbf{A}\left[\begin{array}{c}
\mathbf{q} \\
\boldsymbol{\lambda}
\end{array}\right] \leq \mathbf{b} \\
{\left[\begin{array}{ll}
\mathbf{q}^{T} & \left.\boldsymbol{\lambda}^{T}\right] \text { is binary }
\end{array}\right.}
\end{array}
\end{aligned}
$$

where matrix $A$ and vector $b$ depend on $W$ and $V$, according to constraints (6), (7) and (8). Remark that the optimization vector has been extended to include the linear MSO set selector $\lambda$. This implies that the cost vector is also extended. However, since the purpose of the optimisation is to find the set of optimal sensors, the cost related to the $\boldsymbol{\lambda}$ is set to zero. By doing this, the selected valid MSO sets in the optimisation problem have no effect in the solution cost. Hence, $\boldsymbol{\lambda}$ is regarded as a dummy vector.

\section{INCREMENTAL APPROACH (Rosich et al., 2007)}

Given a sensor configuration $S$, one approach to compute $\Omega_{S}$ is using Algorithm MSO in Krysander et al. (2008). However, the time complexity of this algorithm is exponential with the cardinality of $S$. A more efficient alternative approach, which involves the incremental computation of $\Omega_{S}$, is recalled in this section.

Performing the sensor placement analysis involves the addition of new measurements to the system model. In the structural framework, a sensor will be represented by one single equation denoted as sensor equation $e_{s}$. Without loss of generality, it is assumed that this equation involves one single unknown variable $x_{i} \in X$. Given a set of sensors $S$, the following set is defined $M_{S}=\left\{e_{s} \mid s \in S\right\}$. Thus, $M_{\mathbf{S}}$ is the set of all candidate sensor equations.

An MSO set $\omega$ is a subset of model equations that is structurally over-determined, and no proper subset of $\omega$ is over-determined (Krysander et al., 2008). Given two MSO sets $\omega, \omega^{\prime} \in \Omega_{\mathbf{S}}$ the following equivalence relation is defined:

$$
\omega \sim \omega^{\prime}: \omega \cap M_{\mathbf{S}}=\omega^{\prime} \cap M_{\mathbf{S}}
$$

Based on this equivalence relation, an equivalence class $[\omega]_{S}$ can be defined as

$$
[\omega]_{S}=\left\{\omega^{\prime} \in \Omega_{\mathbf{S}} \mid \omega \sim \omega^{\prime}\right\}
$$

where $S \subseteq \mathbf{S}$ satisfies $M_{S}=\omega \cap M_{\mathbf{S}}$ for any MSO set $\omega \in[\omega]_{S}$. Thus, $[\omega]_{S}$ represents the set of MSO sets that depend on the same set of sensors $S$.

Given a sensor configuration $S, \Omega_{S}$ can be partitioned into the following quotient set $\Omega_{S} / \sim=\left\{[\omega]_{S} \mid \omega \in \Omega_{S}\right\}$. Thus, $\Omega_{S}$ can be computed as:

$$
\Omega_{S}=\bigcup_{S_{i} \subseteq S}[\omega]_{S_{i}} \quad \forall[\omega]_{S_{i}} \in \Omega_{S} / \sim
$$

It is particularly interesting to compute $\Omega_{S}$ as follows:

$$
\Omega_{S}=[\omega]_{S} \cup \bigcup_{S^{\prime} \subset S}[\omega]_{S^{\prime}}
$$

Therefore, forcing a sensor placement search strategy in increasing sensor configuration cost, implies that when evaluating $\Omega_{S}$, all $[\omega]_{S^{\prime}} \forall S^{\prime} \subset S$ will have been computed in a previous iteration since $C\left(S^{\prime}\right)<C(S)$.

Algorithm 2 incrementally solves the optimal sensor placement problem.

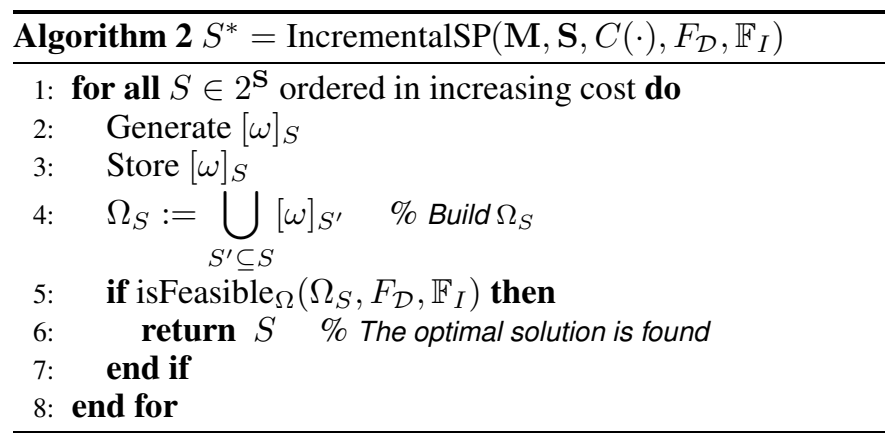

In Step 2 of Algorithm 2, $[\omega]_{S}$ can be computed applying a modified version of Algorithm FindMSO in Krysander et al. (2008). The boolean function isFeasible ${ }_{\Omega}$ verifies the fault diagnosis specifications for a given sensor configuration. This function is implemented based on the Fault Signature Matrix approach recalled in Section 2.

\section{APPLICATION TO A FUEL CELL STACK SYSTEM}

\subsection{Fuel-cell system description}

A PEM (Polymer Electrolyte Membrane) Fuel Cell System model is used to compare the three optimal sensor placement approaches. A model for a PEM Fuel Cell was proposed in Pukrushpan et al. (2004). This model is widely accepted nowadays in the control community as a good representation of the behavior of an actual fuel cell for control purposes. The main components considered in the system (see Figure 1) are the air compressor, the air manifold, the fuel cell stack, the anode manifold and the return manifold. In the model, it is assumed that the temperature is known and constant, since its dynamical behaviour is much more slower than those of the rest of the model.

Relations between model equations and system unknown variables are summarized below. Note that no analytic expression nor parameter are detailed since only the structure of the model is of interest. These relations are derived from the non-linear 


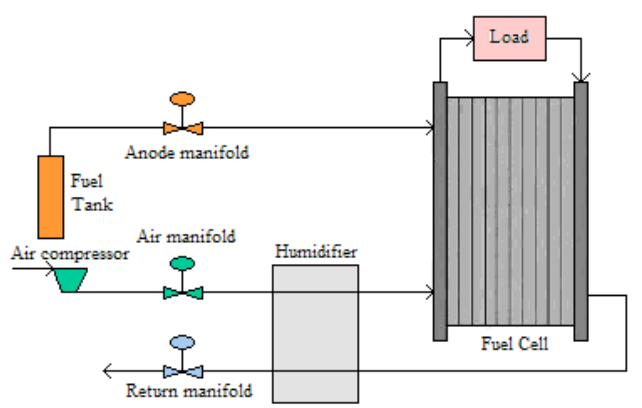

Fig. 1. Fuel Cell Stack System

equations in Pukrushpan et al. (2004) and they are classified according to the system component that they describe:

- Air Supply Compressor:

$e_{1}: f_{1}\left(\omega_{c p}, \tau_{c m}, \tau_{c p}\right)=0$

$e_{2}: f_{2}\left(\tau_{c m}, V_{c m}, \omega_{c p}\right)=0$

$e_{3}: f_{3}\left(\tau_{c p}, \omega_{c p}, p_{s m}, W_{c p}, f_{p_{s m}}\right)=0$

$e_{4}: f_{4}\left(W_{c p}, p_{s m}, \omega_{c p}, f_{p_{s m}}\right)=0$

- Air Supply Manifold:

$e_{5}: f_{5}\left(W_{s m, o u t}, p_{s m}, p_{c a}, f_{W_{s m, o u t}}\right)=0$

$e_{6}: f_{6}\left(W_{c p}, W_{s m, o u t}, f_{p_{s m}}, f_{W_{s m, o u t}}\right)=0$

- Fuel Cell Stack:

$e_{7}: f_{7}\left(W_{c a, \text { out }}, W_{v, \text { inj }}, I_{s t}, W_{s m, \text { out }}, f_{W_{s m, o u t}}, f_{I_{s t}}, f_{n}\right)=0$

$e_{8}: f_{8}\left(W_{a n, i n}, I_{s t}, f_{I_{s t}}, f_{n}\right)=0$

$e_{9}: f_{9}\left(W_{s m, o u t}, p_{c a}, W_{v, \text { inj }}, I_{s t}, p_{a n}, V_{s t}, f_{W_{s m, o u t}}, f_{I_{s t}}\right)=0$

- Anode Manifold: $e_{10}: f_{10}\left(W_{a n, i n}, p_{a n}\right)=0$

- Return Manifold:

$e_{11}: f_{11}\left(W_{c a, \text { out }}, W_{r m, \text { out }}, f_{W_{r m, \text { out }}}\right)=0$

$e_{12}: f_{12}\left(p_{c a}, W_{\text {ca,out }}, W_{\text {rm,out }}, f_{W_{r m, \text { out }}}\right)=0$

Model variables are classified into the following categories:

- Control variables: variables required for control purposes. These variables are already measured.

$V_{c m}$ : Compressor voltage

$W_{c p}$ : Air flow through the compressor

$I_{s t}:$ Stack current

$V_{s t}:$ Stack voltage

- Unmeasurable variables: variables for which sensors are not available.

$\tau_{c m}$ : Compressor motor torque

$\tau_{c p}:$ Load torque

$W_{v, i n j}:$ Humidifier injector flow

- Measurable variables: define all possible sensor locations.

$\omega_{c p}$ : compressor angular speed

$p_{s m}$ : Supply manifold pressure

$W_{\text {sm,out }}$ : Supply manifold exit flow

$p_{c a}$ : Cathode pressure

$W_{\text {ca, out }}$ : Cathode output flow

$p_{a n}$ : Anode pressure

$W_{a n, i n}$ : Anode input flow

$W_{\text {rm,out }}$ : Return manifold exit flow

- System Faults.

$f_{p_{s m}}$ : Compressor fault

$f_{W_{s m, o u t}}$ : Supply manifold fault

$f_{n}$ : Cell fault

$f_{I_{s t}}:$ Fuel Cell Stack fault

$f_{W_{r m, o u t}}$ : Return manifold fault

Each measurable variable has an associated sensor which together constitute the set of candidate sensors. The fault diagnosis specifications to be fulfilled in this application are that all system faults must be detectable and isolable. It has been veri-
Table 1. Sensor cost for each measurable variable

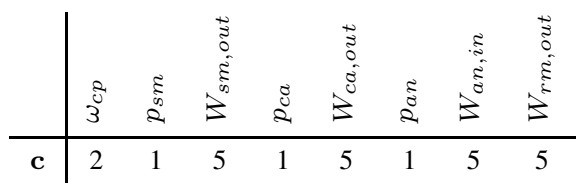

fied that these specifications would be satisfied if all candidate sensors were installed.

\subsection{Optimal Sensor Placement Solution}

Solving the optimal sensor placement problem requires a cost to be associated to each candidate sensor. Consider sensor cost $\mathbf{c}$ in Table 1. Costs are dimensionless and have been assigned according to the ease of installation and the price of their corresponding sensors. The three approaches compute, the same optimal solution, as expected. The optimal sensor configuration is $S^{*}=\left\{p_{c a}, p_{a n}\right\}$ with a cost $C\left(S^{*}\right)=2$.

\section{COMPARATIVE STUDY}

The three approaches to optimal sensor placement are compared in this section. The comparison is based on the results obtained in Section 6, when applied to the Fuel Cell Stack System. Remark that the performance of the three search algorithms depends on different issues such as the number of candidate sensors, their cost, the number of model equations and the number of faults.

\subsection{Performance Issues}

The performance of the three approaches is evaluated in this section. All algorithms have been run on a dual core 2.4 $\mathrm{GHz}$ laptop computer running Windows 7, 32 bit version. All simulation running times are mean values.

Regarding the heuristic approach, Algorithm 1 has been implemented in MATLAB. Given a set of 8 candidate sensors, the sensor placement algorithm must search a solution among $2^{8}=256$ potential sensor configurations. However, Algorithm 1 just needs to traverse 36 sensor configurations. Of these, the fault diagnosis specifications are only verified against 10 sensor configurations. After all this procedure the solution is found in 0.12 seconds.

The BILP approach requires the previous computation of the complete set of MSO sets $\Omega_{\mathbf{S}}$. Algorithm MSO in (Krysander et al., 2008) has been implemented in MATLAB. It takes 94.90 seconds to compute up to $9039 \mathrm{MSO}$ sets. Once $\Omega_{\mathrm{S}}$ is computed, matrices $V$ and $W$ can be easily obtained.

The BILP formulation (9) involves up to 9064 constraints and has been solved using ILOG CPLEX Optimization Studio (IBM, 2010). The solution is found in 12.43 seconds, $\mathbf{q}^{*}=\left[\begin{array}{llllllll}0 & 0 & 0 & 1 & 0 & 1 & 0 & 0\end{array}\right]^{T}$. This corresponds to the sensors measuring variables $p_{c a}$ and $p_{a n}$, as expected. Moreover, the solution includes a set of selected MSO sets through vector $\boldsymbol{\lambda}$, that depends on those sensors and guarantee fault detectability and isolability.

MATLAB is another environment that could solve the BILP formulation (9). However, MATLAB can not be applied in this case since it does not support the large size of matrices $A$ and $b$ in (4). 
Regarding the incremental approach, Algorithm 2 has also been implemented in MATLAB. It just needs to check 7 out of 256 potential sensor configurations, and the total amount of MSO sets computed to check all of them is 50 out of 9039 . In the end, the full set of MSO sets corresponding to the optimal sensor configuration contains $32 \mathrm{MSO}$ sets (i.e., $\left|\Omega_{S^{*}}\right|=32$ ). After all this procedure the solution is found in 0.32 seconds.

\subsection{Discussion}

From the point of view of efficiency, the most time demanding task is the computation of $\Omega_{\mathrm{S}}$. Thus, this is a severe drawback for the BILP approach. In this case, even the optimal sensor configuration search alone takes much more time than the other two approaches, although CPLEX optimization tools are applied. The incremental approach performance is good. However, since the search is monotonically increasing on the sensor configuration cost, as a solution involves a sensor configuration with a higher cost, the computation time is expected to increase significantly. Finally, the heuristic approach is the most efficient. Above all, this is due to the fact that it does not require the computation of MSO sets. Also, in this approach the optimal search is expected to perform better in average than in the incremental approach.

In this paper, fault detectability and isolability have been considered as fault diagnosis specifications. If other specifications were to be satisfied, the applicability of one approach or another would depend on whether they were stated on the set of MSO sets or on the model equations (heuristic approach). In the case of being stated on the set of MSO sets, the incremental approach offers more flexibility, since the BILP approach requires the formulation of the specifications as a set of linear inequalities.

Recall from Section 2 that candidate sensor faults are not considered. Extensions that consider candidate sensor faults exist for the incremental approach (Rosich et al., 2007) as well as for the BILP approach (Rosich et al., 2009). Up to now this problem has not been solved for the heuristic approach. Therefore, in this case, the incremental approach would presumably be preferable over the BILP approach.

\section{CONCLUSIONS}

In this paper, three approaches to optimal sensor placement have been briefly recalled. The main contribution of this work is a comparative study on the performance of these approaches when applied to a fuel cell stack system. The overall conclusion is that each approach offers benefits but also drawbacks depending on the criterium being evaluated. Choosing one approach or another will depend on the stated application specifications.

In model-based fault diagnosis, diagnosis is basically performed based on the response of residual generators, which are derived from the model equations. When the model includes nonlinearities, deriving a residual generator can become a difficult or even a practically infeasible task. In this paper, this issue has been ignored. However, any of the three approaches could be adapted to take into account this issue in the sensor placement analysis phase, by following the causality framework introduced in Rosich et al. (2012). Then, the solution obtained from the sensor placement analysis would guarantee a set of particularly easy computable residual generators.

\section{REFERENCES}

Dulmage, A.L. and Mendelsohn, N.S. (1958). Covering of bipartite graph. Canada J. Math, 10, 527-534.

Fijany, A. and Vatan, F. (2006). A new efficient algorithm for analyzing and optimizing the system of sensors. In Proc. 2006 IEEE Aerospace Conference. Big Sky, Montana, USA.

IBM (2010). IBM ILOG CPLEX optimization studio 12.2. http://www-01.ibm.com/software/integration/ optimization/cplex-optimization-studio/.

Krysander, M. (2006). Design and Analysis of Diagnosis Systems Using Structural Analysis. Ph.D. thesis, Linköping Univ., Linköping, Sweden.

Krysander, M., Åslund, J., and Nyberg, M. (2008). An efficient algorithm for finding minimal over-constrained sub-systems for model-based diagnosis. IEEE Trans. Syst., Man, Cybern. A, 38(1).

Krysander, M. and Frisk, E. (2008). Sensor placement for fault diagnosis. IEEE Trans. Syst., Man, Cybern. A, 38(6), 13981410.

Nejjari, F., Sarrate, R., and Rosich, A. (2010). Optimal sensor placement for fuel cell system diagnosis using bilp formulation. In 18th Mediterranean Conference on Control and Automation, 1296-1301. Marrakech, Morocco.

Ploix, S., Yassine, A.A., and Flaus, J.M. (2008). An improved algorithm for the design of testable subsystems. In Proc. of 17 th IFAC World Congress. Seoul, Korea.

Pukrushpan, J.T., P., H., and Stefanopoulou, A.G. (2004). Analysis for automotive fuel cell systems. Transactions of the ASME, 126, 14-25.

Pulido, B. and Gonzalez, C.A. (2004). Possible conflicts: a compilation technique for consistency-based diagnosis. IEEE Trans. Syst., Man, Cybern. B, 34(5), 2192-2206.

Rosich, A., Sarrate, R., and Nejjari, F. (2009). Optimal sensor placement for FDI using binary integer linear programming. In 20th International Workshop on Principles of Diagnosis (DX-09). Stockholm, Sweden.

Rosich, A., Sarrate, R., Puig, V., and Escobet, T. (2007). Efficient optimal sensor placement for model-based FDI using and incremental algorithm. In Proc. 46th IEEE Conference on Decision and Control, 2590-2595. New Orleans, USA.

Rosich, A., Yassine, A.A., and Ploix, S. (2010). Efficient optimal sensor placement for structural model based diagnosis. In 21th International Workshop on Principles of Diagnosis (DX-10). Portland, USA.

Rosich, A., Frisk, E., Åslund, J., Sarrate, R., and Nejjari, F. (2012). Fault diagnosis based on causal computations. IEEE Trans. Syst., Man, Cybern. A, 42(2), 371-381.

Sarrate, R., Puig, V., Escobet, T., and Rosich, A. (2007). Optimal sensor placement for model-based fault detection and isolation. In Proc. 46th IEEE Conference on Decision and Control, 2584-2589. New Orleans, USA.

Travé-Massuyès, L., Escobet, T., and Olive, X. (2006). Diagnosability analysis based on component supported analytical redundancy relations. IEEE Trans. Syst., Man, Cybern. A, 36(6), 1146-1160.

Wosley, L.A. (1998). Integer Programming. John Wiley \& Sons, New York, USA.

Yassine, A.A., Ploix, S., and Flaus, J.M. (2008). A method for sensor placement taking into account diagnosability criteria. Int. J. Appl. Math. Comput. Sci., 18(4), 497-512. 